Hobbs notes. "As well as providing the instrument, we provide the methodology and buffers, but we do specify you have to use that method to get the full support. It's a little bit different from the normal research instrument approach, but we thought there was a need for that and it seems to be accepted."

Beckman is also currently commercializing an innovative system of protein partitioning using affinity fractionation to decrease the unwanted complexity of protein mixtures before analysis. The aim is to remove not just very abundant proteins, for example serum albumin from blood plasma, but also other proteins that are already well characterized. The firm claims that up to $95 \%$ of the proteins in a cell lysate can be removed before the full fractionation stage with less risk than other purification procedures of losing the proteins you're interested in. "There's a growing interest in what might be going away with these large-abundance proteins — albumin is a binding protein, and possibly some interesting proteins go with it," Hobbs notes.

Several big equipment producers have teamed up with smaller specialist firms to include cutting-edge reagents or media in application kits for their automated systems. Tecan in Männedorf, Switzerland, recently signed a licensing agreement to deploy the paramagnetic beads developed by Dynal Biotech in Oslo, Norway, on its Freedom EVO liquid-handling platform (see 'Attracting attention', page 796). The Robopop protein purification kits from Novagen in Madison, Wisconsin, can also be used on
Tecan's workstation and on the MultiPROBE liquid-handling workstation from PerkinElmer in Boston, Massachusetts.

Caliper Life Sciences in Hopkinton, Massachusetts, has integrated into its Sciclone ALH3000 liquid-handling workstation a new column technology developed by PhyNexus based in San Jose, California (see 'Smallscale separation', page 795). The combination allows researchers to purify and enrich small quantities of up to 96 engineered proteins in as little as 15 minutes.

The market for protein purification systems has changed in the past six months, notes Mark Roskey, vice-president of marketing at Caliper, with more groups getting involved in larger-scale protein purification. "It's not at the industrial scale, but regular pharma and biotech R\&D people are now trying to purify proteins in a more parallel situation. A lot of this stems from having all the genes and working with huge numbers of them to develop new drugs," he says.

\section{Analysis and optimization}

To maintain the benefits of high-throughput separation and purification, the proteins of interest must be able to pass smoothly into the next stage of the process. "Once you've got a relatively pure protein you need to determine whether it is pure, so

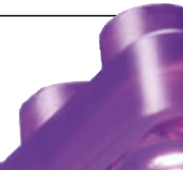

$=\lim _{m}$

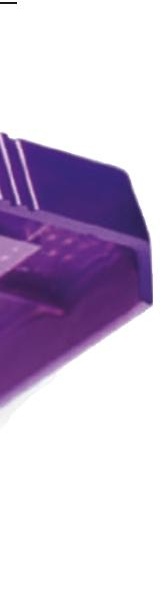

The LabChip90 from Caliper

Life Sciences.

there's issues with analysis as well," says Roskey. A common analysis method is SDSpolyacrylamide gel electrophoresis "but we feel that that is a bottleneck", Roskey adds.

Although the established protocols of macroscale gel electrophoresis are being successfully automated (see 'Automation in two dimensions', below), many users are turning instead to microfluidic and lab-on-a-chip solutions. In January, Caliper launched the Protein Express Assay for its LabChip 90 automated electrophoresis system.

"It's a microfluidic replacement for SDS-PAGE that automates the whole process," Roskey says. "Rather than putting samples on a gel, you get them off a multiwell plate, and it does integrated separation,

\title{
AUTOMATION IN TWO DIMENSIONS
}

Microfluidic systems have taken over from two-dimensional (2D) gel electrophoresis techniques in some areas of protein separation and analysis, but the established methods are far from dead. Even though they can be slower and messier, the tried and tested 2D protocols still offer some advantages, especially if automation can take out most of the hassle.

"Some people will be claiming otherwise, but I think 2D still has the best levels of sensitivity when you're looking at complex samples," says Paul Orange, senior product manager at NextGen Sciences in Cambridge, UK. "With 2D you're seeing resolution of 2,000-3,000 spots on a gel that's quite a lot of information, but people understand it. Also 2D is a very accessible technology - you can go and buy equipment relatively cheaply and get started if you're looking for a proteomics approach."

In 2003, NextGen launched the first fully automated 2D electrophoresis platform, called a2DE. The firm has now brought out a spin-off system called the a2DEoptimizer which can improve 2D separations on a variety of commercial platforms. "We've not tried to reinvent the wheel," says Orange. "We know what people are using, and know that they have lots of data and experience, but we can help them out by automating key aspects of the process."

The heart of the a2DEoptimizer is automated gel casting, allowing researchers to create customized gradient gel profiles with a minimum of fuss. "Everyone knows casting a gradient gel gives you far superior spot resolution, separation and definition, but the problem is that these gradients can be quite tricky to pour, especially the more exotic ones," Orange says. "There's a very small number of people who can get good reproducible gels when they're pouring gradients. As we see 2D going forward, people are dealing with very small amounts of sample. They've got one or two gels they can run so they have to get the best data they can out of there."

The system also has integrated power packs that can focus samples at high voltages and reduce the time for a separation run. And it has real-time monitoring of the electrical profile. "That's of particular importance, because when you're dealing with new samples, it's very important to look at the electrical profile and tell whether you've got some kind of issue with salt content or protein content," Orange notes. "What we're doing is enabling people to get better data out, and also analyse what's going on in their system." T.C. 